\title{
PREPARAÇÃO DE DERIVADOS DO LAPACHOL EM MEIO ÁCIDO E EM MEIO BÁSICO: UMA PROPOSTA DE EXPERIMENTOS PARA A DISCIPLINA DE QUÍMICA ORGÂNICA EXPERIMENTAL
}

Ticiano Pereira Barbosa* e Hermes Diniz Neto

Faculdade de Ciências Médicas da Paraíba, Campus II, Praça de São Francisco, 16, 58010-650 João Pessoa - PB, Brasil

Recebido em 12/5/12; aceito em 31/8/12; publicado na web em 6/2/13

PREPARATION OF LAPACHOL DERIVATIVES IN ACID AND BASE MEDIA: PROPOSED EXPERIMENTS FOR ORGANIC CHEMISTRY LABORATORY CLASSES. In this educational paper we describe the extraction of lapachol from its natural source according to acid-base concepts in organic chemistry and the use of its derivatives $\beta$-lapachone and hydroxy-hydrolapachol to exemplify intramolecular cyclization, carbocation stability, Michael addition reaction and chromatography. The experiments were performed during three different undergraduate organic chemistry laboratory classes using low cost material, while avoiding color reagents for TLC visualization, as well as small-scale column chromatography to isolate the mixture of lapachol and $\beta$-lapachone.

Keywords: lapachol; acid-base concepts; chromatography.

\section{INTRODUÇÃO}

O uso do lapachol (1) no Brasil conta com uma longa história, culminando no seu uso como anticâncer através do Laboratório Farmacêutico do Estado de Pernambuco (LAFEPE). ${ }^{1}$ O lapachol (1) é uma naftoquinona isolada de uma fonte natural, o cerne do lenho de árvores da família do ipê (Tabebuia spp., Bignoniaceae), com um rendimento variado entre 1 e $7 \%$ em massa, dependendo da espécie e de outros fatores como a região e a sazonalidade. ${ }^{2} \mathrm{O}$ conhecimento deste produto natural data de $1858^{3}$ e seu estudo químico possui notório volume de publicações devido aos trabalhos pioneiros de S. C. Hooker, ${ }^{4,5}$ bem como publicações póstumas feitas por L. F. Fieser, apresentando uma série de artigos contendo resultados de trabalhos de Hooker. ${ }^{6}$

Devido ao seu caráter ácido, com pKa determinado através de titulações $\mathrm{pH}$-métricas e espectrofotométricas ${ }^{7} \mathrm{em}$ aproximadamente 6,0, o lapachol (1) pode ser prontamente extraído de sua fonte natural quando em contato com uma solução alcalina. ${ }^{8}$ Em sua forma ácida, apresenta-se como um sólido de cor amarelada e insolúvel em água, entretanto, o seu sal (base conjugada) (2) apresenta grande solubilidade em água e uma coloração vermelha. Assim, uma solução de $\mathrm{Na}_{2} \mathrm{CO}_{3}$ ou $\mathrm{NaHCO}_{3}$ pode ser empregada para tal extração, como exemplificado no Esquema 1. Podemos escrever ainda mais duas estruturas canônicas que contribuem para a estabilidade da base conjugada 2.
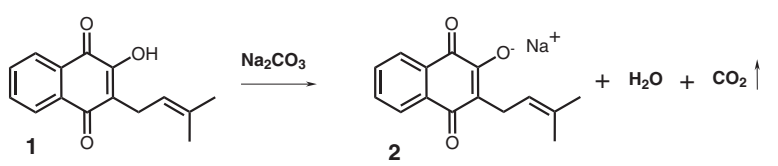

Esquema 1. Reação de ionização do lapachol (1) em solução de carbonato de sódio

Outra naftoquinona natural que possui destaque é a $\beta$-lapachona (3). Esse isômero constitucional do lapachol (1), também presente em algumas espécies da família Bignoniaceae, possui grande interesse científico devido ao seu perfil farmacológico. ${ }^{8}$ Em 1882, Paternò obteve sinteticamente, pela primeira vez, a $\beta$-lapachona (3) através de um tratamento ácido do lapachol $(\mathbf{1})^{9} \mathrm{e}$, subsequentemente, Hooker determinou sua estrutura e condições para a formação deste produto

*e-mail: ticianopcpb@hotmail.com natural, além da $\alpha$-lapachona (4), uma naftoquinona isomérica de origem natural, ${ }^{10}$ de acordo com o Esquema 2.

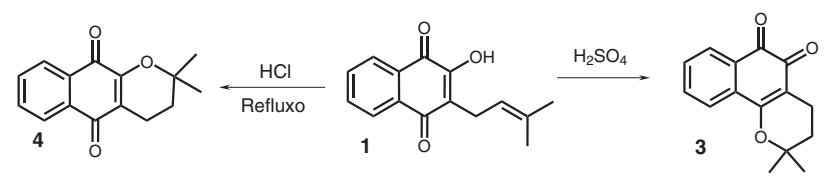

Esquema 2. Formação $\beta$-lapachona (3) e da $\alpha$-lapachona (4) a partir do lapachol (1)

$\mathrm{O}$ uso de $\mathrm{H}_{2} \mathrm{SO}_{4}$ concentrado fornece exclusivamente a $\beta$-lapachona (3), sendo esta seletividade estudada por Ettlinger. ${ }^{11}$ No trabalho em questão, a formação exclusiva de $\mathbf{3}$ está relacionada à sua basicidade. A $\beta$-lapachona (3) se mostrou muito mais básica que seu isômero $\alpha$, já que sua forma ácida 6 apresentou $\mathrm{pKa}=-3,45$, valor este maior em quase três unidades em relação à $\alpha$-lapachona $(\mathbf{4})(\mathrm{pKa}$ $=-6,35) \cdot{ }^{11}$ Após a diluição do meio reacional com água, o $\beta$-cátion 6 libera rapidamente $\mathbf{3}$ e esta precipita. A formação dos produtos cíclicos é bastante favorecida em meio ácido devido à formação do carbocátion terciário $\mathbf{5}$, após uma reação de adição à ligação dupla da cadeia lateral (Esquema 3).

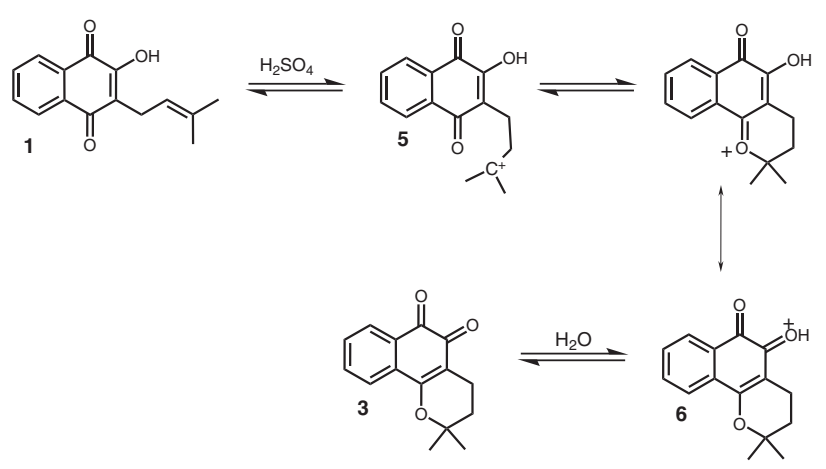

Esquema 3. Ciclização do lapachol (1) para formação da $\beta$-lapachona (3)

Em trabalho realizado por Hooker, a $\beta$-lapachona (3) foi submetida a uma solução alcalina sob aquecimento. O produto obtido para a reação foi denominado por ele de hidroxi-hidrolapachol (7), Esquema 4 , resultante da abertura do anel heterocíclico pela base utilizada. ${ }^{12}$ 

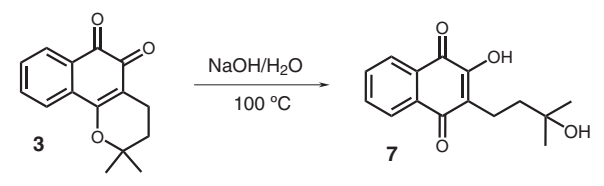

Esquema 4. Abertura do anel heterocíclico de 3 com base sob aquecimento

Um mecanismo proposto para a reação é a abertura do anel heterocíclico através de uma adição do tipo Michael com ataque nucleofílico do íon hidróxido ao sistema $\alpha, \beta$-insaturado da naftoquinona, seguido por uma eliminação. Alguns estudos corroboram esta proposta mecanística, quando da obtenção de adutos da $\beta$-lapachona após ataque nucleofílico do aminoácido cisteína e do 2-mercaptoetanol ao sistema $\alpha, \beta$-insaturado da $\beta$-lapachona (3). ${ }^{13,14}$

Esta proposta de trabalho com o lapachol se torna viável por ser um produto natural obtido em quantidade satisfatória (gramas) de sua fonte natural, utilizando recursos de baixo custo e com potencial diversidade para aulas práticas em Química Orgânica, como a exemplificação de diversas reações orgânicas através da síntese de naftoquinonas derivadas deste produto natural, ${ }^{15,16}$ bem como da modificação estrutural da $\beta$-lapachona. ${ }^{17}$ Além da possibilidade de explorar a reatividade das naftoquinonas em aulas práticas, pode-se também utilizar tais compostos para demonstrar as técnicas de cromatografia em camada delgada (CCD) e cromatografia em coluna (CC).

A CCD é uma técnica simples e de execução rápida, sendo uma ferramenta eficaz de análise qualitativa para avaliação de uma amostra simples. A técnica possui várias finalidades como: avaliação do número de componentes de uma mistura, determinação da identidade de uma amostra por comparação com um padrão, ser utilizada também para monitoramento do progresso de uma reação química ou escolha de um solvente apropriado para uma separação por CC e seu monitoramento durante a separação. ${ }^{18}$ Entretanto, é comum a utilização de reveladores químicos ou indicadores de fluorescência em luz ultravioleta para que os compostos orgânicos sejam visualizados nas placas cromatográficas. Uma vez que as naftoquinonas se apresentam na forma de cristais em diversas colorações, tais como, amarela, vermelha e laranja, há uma grande praticidade na utilização destes compostos em cromatografia, pois dispensam o uso de reveladores químicos, indicadores de fluorescência ou câmara de ultravioleta, tornando os experimentos práticos, simples e de baixo custo. O emprego destes compostos torna as técnicas de CCD e CC visualmente didáticas, visto que a separação dos componentes da mistura pode ser observada na forma de manchas distintas durante $o$ desenvolvimento dos cromatogramas. Outra proposta deste trabalho é a utilização da técnica de CC em escala reduzida, ${ }^{19}$ minimizando as quantidades do solvente orgânico e da sílica gel empregados.

\section{PARTE EXPERIMENTAL}

\section{Primeira aula: extração e purificação do lapachol (1)}

A extração de 1 foi realizada de acordo com a metodologia proposta por Ferreira. ${ }^{20}$ Aproximadamente $200 \mathrm{~g}$ de serragem de ipê foram colocados em um béquer de $2 \mathrm{~L}$ e, em seguida, adicionou-se 1 $\mathrm{L}$ de uma solução $1 \%$ de $\mathrm{Na}_{2} \mathrm{CO}_{3}$. A mistura foi deixada em repouso por $45 \mathrm{~min}$, com agitação ocasional com bastão de vidro. A solução extratora, de coloração avermelhada, foi filtrada sobre gaze. Ao filtrado adicionou-se vagarosamente uma solução de $\mathrm{HCl} 6 \mathrm{M}$ até a solução vermelha tornar-se amarela. Nesta etapa já é possível observar a formação do lapachol (1) na forma de um precipitado amarelo. $\mathrm{O}$ lapachol (1) precipitado foi coletado através de uma filtração simples (aproximadamente $30 \mathrm{~min}$ ), podendo ser feita filtração a vácuo. Após a filtração, o material foi seco em estufa a $80^{\circ} \mathrm{C}$ por $1 \mathrm{~h}$, obtendo-se
2,3 g do produto. A purificação do lapachol (1) foi executada, com auxílio do monitor da disciplina, por cromatografia em coluna de sílica gel 60 (63-200 mm, 70-230 Mesh ASTM) (20 g) utilizando coluna de vidro $(\mathrm{h}=30 \mathrm{~cm}$ e $\varnothing=3,0 \mathrm{~cm})$ e sistema eluente acetato de etila/ hexano (3:7) (250 mL). Após evaporação do solvente foram obtidos $1,3 \mathrm{~g}$ de lapachol (1) na forma de cristais amarelos. P.F.: $139-140^{\circ} \mathrm{C}$. (lit. $\left.139,5-140,2^{\circ} \mathrm{C}\right) .^{1}$

\section{Segunda aula: síntese da $\beta$-lapachona (3) e do hidroxi-hidrola- pachol (7)}

\section{$\beta$-lapachona (3)}

Um béquer de $50 \mathrm{~mL}$ contendo $484 \mathrm{mg}$ do lapachol (1) foi colocado em banho de gelo e adicionaram-se lentamente 5,0 mL de $\mathrm{H}_{2} \mathrm{SO}_{4}$ concentrado. A mistura reacional foi mantida por $15 \mathrm{~min}$ no banho de gelo com agitações a cada 5 min. Após o término da reação, a mistura foi vertida em $150 \mathrm{~mL}$ de água destilada gelada e o precipitado, de coloração laranja, foi filtrado em papel previamente pesado, lavado com água destilada e seco em estufa a $80^{\circ} \mathrm{C}$ por $1 \mathrm{~h}$. Depois de seco e pesado, o material forneceu $339 \mathrm{mg}$ (Rend. $=70 \%$ ). P.F.: $154-156^{\circ} \mathrm{C}$. (lit. 154,5 a $155,4{ }^{\circ} \mathrm{C}$ )..$^{21}$

\section{Hidroxi-hidrolapachol (7)}

Em um béquer de $100 \mathrm{~mL}$ contendo $242 \mathrm{mg}$ de 3 adicionaram-se $30 \mathrm{~mL}$ de uma solução $5 \%$ de $\mathrm{NaOH}$. A mistura foi aquecida em chapa aquecedora e agitada até a ebulição e total dissolução de $\mathbf{3}$, formando uma solução de cor vermelha. Após 2-3 min de ebulição, a mistura foi resfriada e uma solução de $\mathrm{HCl} 6 \mathrm{M}$ foi gotejada até a mudança de coloração para amarela, indicando a formação do hidroxi-hidrolapachol (7). Foi realizada uma extração líquido-líquido em funil de separação de $125 \mathrm{~mL}$ com $30 \mathrm{~mL}$ de acetato de etila. A fase orgânica contendo 7 foi coletada, seca com $\mathrm{Na}_{2} \mathrm{SO}_{4}$ anidro e após evaporação do solvente foram obtidos $161 \mathrm{mg}$ de um sólido amarelo (Rend. $=62 \%$ ). P.F.: $125-126^{\circ} \mathrm{C}$ (lit. $\left.125-125,5^{\circ} \mathrm{C}\right) .{ }^{22}$

\section{Terceira aula: uso das naftoquinonas em cromatografia}

\section{Cromatografia em camada delgada $(C C D)$}

Para a realização da técnica de CCD utilizou-se uma cromatofolha de sílica gel 60 do fabricante Macherey-Nagel, cortada com estilete nas dimensões 5 x $10 \mathrm{~cm}$. Foram marcados 4 pontos de aplicação com espaçamento de $0,5 \mathrm{~cm}$ das bordas laterais da placa e $1 \mathrm{~cm}$ entre cada um dos pontos. Abaixo dos pontos de aplicação foram grafadas as letras L (lapachol), B ( $\beta$-lapachona), H (hidroxi-hidrolapachol) e M (mistura de todos). Uma ponta de espátula de cada uma dos compostos foi adicionada em 3 frascos distintos e dissolvida com $1 \mathrm{~mL}$ de acetato de etila. As amostras foram aplicadas com capilar de vidro e a placa foi colocada no interior de uma cuba, previamente saturada, para a eluição com acetato de etila/n-hexano (3:7). Após a eluição, foram calculados os fatores de retenção das manchas observadas.

\section{Cromatografia em coluna com escala reduzida}

Foram pesados, em um mesmo béquer de $50 \mathrm{~mL}, 10 \mathrm{mg}$ de $\mathbf{1}$ e $10 \mathrm{mg}$ de 3. Para a separação em escala reduzida foi utilizada pipeta Pasteur com ponta cortada $(\mathrm{h}=15 \mathrm{~cm}$ e $\varnothing=7 \mathrm{~mm}$ ). Um pedaço de algodão foi colocado no fundo da pipeta, que foi adaptada a um suporte universal. Em seguida, foram pesados, em um béquer de $50 \mathrm{~mL}, 2 \mathrm{~g}$ de sílica gel 60 com tamanho de partícula 63-200 mm (70-230 Mesh ASTM) e adicionados $5 \mathrm{~mL}$ da fase móvel acetato de etila/n-hexano (3:7) para preparação da coluna. Após a compactação da coluna, a mistura de 1 e 3 foi dissolvida com $0,5 \mathrm{~mL}$ da fase móvel e transferida para o topo da coluna com auxílio de conta-gotas (ou pipeta Pasteur). Após o volume total da mistura atingir o nível superior da coluna de 
sílica e esta adsorver os componentes da mistura, completou-se o volume da coluna com a fase móvel. As frações foram coletadas em 4 tubos de ensaio e, em seguida, analisadas por CCD.

\section{RESULTADOS E DISCUSSÃO}

O tempo de extração do lapachol (1) de sua fonte foi adequado para a realização da aula, sendo possível obter $1,3 \mathrm{~g}$ do produto purificado a partir de $200 \mathrm{~g}$ de serragem de ipê, quantidade suficiente para realizar os demais experimentos propostos. $\mathrm{O}$ emprego do $\mathrm{Na}_{2} \mathrm{CO}_{3}$ em lugar de bases mais fortes evita a extração de compostos ácidos mais fracos que o lapachol. Durante a extração, o professor poderá explicar conceitos de ácidos e bases em química orgânica e mostrar o Esquema 1 para compreensão do caráter ácido do lapachol (1). Em uma análise intrínseca da base conjugada 2, é possível escrever ainda mais duas estruturas canônicas significativas para tal íon, evidenciando sua relativa estabilidade. Durante o procedimento de secagem na estufa, o professor deve explicar o equilíbrio ácido-base entre 2 e o HCl, destacando a formação do lapachol como ácido mais fraco e a pouca solubilidade do mesmo em água, de acordo com sua estrutura. Pode-se também relatar a importância dos produtos naturais como fonte de medicamentos. A purificação de $\mathbf{1}$ feita por cromatografia em coluna trata-se de uma rápida filtração sobre gel de sílica para separar o produto de resinas e fibras de celulose.

$\mathrm{O}$ emprego de $\mathrm{H}_{2} \mathrm{SO}_{4}$ concentrado é necessário para obtenção exclusiva da $\beta$-lapachona (3), com rendimento satisfatório para prosseguir na preparação do hidroxi-hidrolapachol (7). O professor deve apresentar o mecanismo da reação de formação da $\beta$-lapachona (3) de acordo com o Esquema 3. Isso poderá ser feito durante o tempo de secagem do material na estufa. Já a reação de abertura do anel, como já mencionado, ocorre a partir de um ataque nucleofílico do íon hidróxido ao sistema $\alpha, \beta$-insaturado da naftoquinona, através de uma adição tipo Michael 1,4 para a formação do aduto $\mathbf{8}$, seguida de eliminação para a formação do alcóxido terciário 9. Após etapas de transferência de prótons (prototropismo) e acidificação do meio, o produto precipita, como ilustrado no Esquema 5.

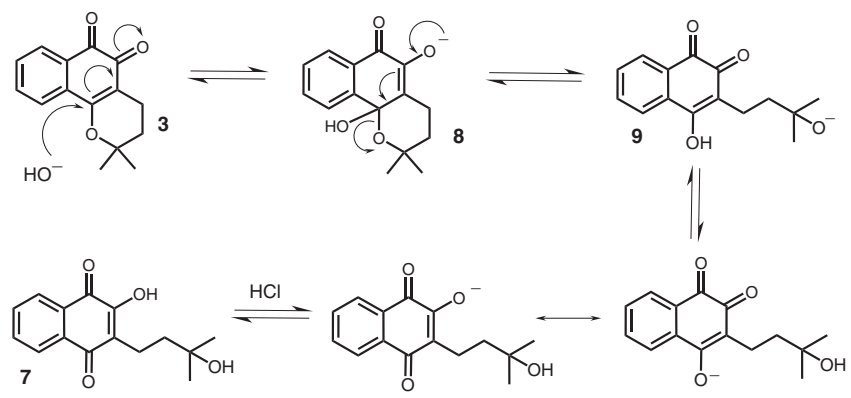

Esquema 5. Mecanismo proposto para a formação do hidroxi-hidrolapachol (7)

O professor deve relacionar as características estruturais das naftoquinonas com o comportamento das mesmas na CCD, abordando temas como forças intermoleculares e polaridade dos compostos através de vetores momento dipolar para explicar os diferentes fatores de retenção observados para as naftoquinonas. Os valores listados na Tabela 1 foram obtidos após 3 determinações.

Para reaproveitamento da placa, após o cálculo dos fatores de retenção, as mesmas foram colocadas em uma cuba contendo metanol para "limpeza". O metanol consegue eluir as três naftoquinonas até o limite da linha de frente do solvente, deixando a placa pronta para reutilização durante a comparação das frações coletadas na cromatografia em coluna. A separação de $\mathbf{1}$ e $\mathbf{3}$ em coluna com escala
Tabela 1. Fatores de retenção calculados para as naftoquinonas utilizadas

\begin{tabular}{ll}
\hline Composto & Fator de retenção $\left(\mathrm{R}_{\mathrm{f}}\right)^{*}$ \\
\hline lapachol & $1^{\mathrm{a}}=0,55 / 2^{\mathrm{a}}=0,56 / 3^{\mathrm{a}}=0,56$ \\
$\beta$-lapachona & $1^{\mathrm{a}}=0,35 / 2^{\mathrm{a}}=0,34 / 3^{\mathrm{a}}=0,35$ \\
hidroxi-hidrolapachol & $1^{\mathrm{a}}=0,20 / 2^{\mathrm{a}}=0,19 / 3^{\mathrm{a}}=0,20$ \\
\hline
\end{tabular}

*Três determinações dos fatores de retenção.

reduzida foi realizada de maneira rápida e com uso $50 \mathrm{~mL}$ da fase móvel, podendo-se visualizar duas bandas distintas ao longo do desenvolvimento do cromatograma. As duas primeiras frações coletadas continham apenas $\mathbf{1}$, na terceira não havia nenhum dos compostos e na quarta foi coletado apenas 3, após análise por CCD. Existem páginas na internet que demonstram como montar uma coluna para cromatografia. $^{23}$

\section{CONCLUSÕES}

A aplicação das técnicas descritas durante as aulas da disciplina Química Orgânica Experimental favoreceu a compreensão dos estudantes acerca dos temas abordados. Assim, apresentamos uma continuidade do uso do lapachol (1), proposto inicialmente por Ferreira, ${ }^{20}$ como recurso didático para aulas experimentais em Química Orgânica, com um tempo máximo de 3 h de duração em cada aula. Podendo ser abordados os temas: ácidos e bases, reações de adição a alcenos, estabilidade de carbocátions, reação de adição de Michael, CCD e CC com escala reduzida, tendo como uma das vantagens o baixo custo dos materiais empregados nas aulas.

\section{MATERIAL SUPLEMENTAR}

No material suplementar, disponível gratuitamente em arquivo PDF em http://quimicanova.sbq.org.br, encontram-se fotografias do cromatograma obtido por CCD e da pipeta de Pasteur utilizada para CC.

\section{AGRADECIMENTOS}

Ao Diretor Geral da Faculdade de Ciências Médicas da Paraíba, O. B. Gama, e à coordenadora do curso de Farmácia, Profa. M. de F. S. Trindade, por incentivarem a realização deste trabalho. Às técnicas do Laboratório de Química Orgânica, S. R. de O. Gonzaga e E. S. Sousa, pela organização do laboratório e indispensável auxílio durante a execução das aulas práticas nas turmas de Farmácia.

\section{REFERÊNCIAS}

1. Araújo, E. A.; Alencar, J. R. B.; Rolim Neto, P. J.; Rev. Bras. Farmacogn. 2002, 12, 57.

2. Burnett, A. R.; Thomson, R. H.; J. Chem. Soc. C 1967, 2100.

3. Arnaudon, G.; Compt. Rend. 1858, 46, 1152.

4. Hooker, S. C.; J. Chem. Soc. 1892, 61, 611.

5. Hooker, S. C.; J. Chem. Soc. 1896, 69, 1355.

6. Hooker, S. C.; J. Am. Chem. Soc. 1936, 58, 1163.

7. Ossowski, T.; Goulart, M. O. F.; Abreu, F. C.; Sant'ana, A. E. G.; Miranda, P. R. B.; Costa, C. O.; Liwo, A.; Falkowski, P.; Zarzeczanska, D.; J. Braz. Chem. Soc. 2008, 19, 175.

8. Da Silva, M. N.; Ferreira, V. F.; De Souza, M.; Quim. Nova 2003, 26, 407.

9. Paternò, E.; Gazz. Chim. Ital. 1882, 12, 337.

10. Thomson, R. H.; Naturally Occurring Quinones, $2^{\text {nd }}$ ed.; Academic Press: New York, 1971, chap. 4. 
11. Ettlinger, M. G.; J. Am. Chem. Soc. 1950, 72, 3090.

12. Hooker, S. C.; J. Am. Chem. Soc. 1936, 58, 1168.

13. Neder, K.; Marton, L. J.; Liu, L. F.; Frydman, N.; Cell. Mol. Biol. 1998, 44,465 .

14. Oliveira-Brett, A. M.; Goulart, M. O. F.; Abreu, F. C.; Bioelectrochemistry 2002, 56, 53.

15. Barbosa, T. P.; Camara, C. A.; Silva, T. M. S.; Martins, R. M.; Pinto, A. C.; Vargas, M. D.; Bioorg. Med. Chem. 2005, 13, 6464.

16. Silva, T. M. S.; Camara, C. A.; Barbosa, T. P.; Soares, A. Z.; Da Cunha, L. C.; Pinto, A. C.; Vargas, M. D.; Bioorg. Med. Chem. 2005, 13, 193.

17. Ferreira, S. B.; Gonzaga, D. T. G.; Santos, W. C.; Araújo, K. G. L.; Ferreira, V. F.; $R V q$ 2010, 2, 140.
18. Silva, R. S.; Ribeiro, C. M. R.; Borges, M. N.; Blois, G. S. O.; Quim. Nova 2009, 32, 2234

19. Pavia, D. L.; Lampman, G. M.; Kriz, G. S.; Engel, R. G.; A Small-scale Approach to Organic Laboratory Techniques, $3^{\text {rd }}$ ed.; Brooks/Cole: Belmont, 2010, chap. 19.

20. Ferreira, V. F.; Química Nova na Escola 1996, nº. 4, 35.

21. Alves, G. M. C.; Rolim, L. A.; Rolim Neto, P. J.; Leite, A. C. L.; Brondani, D. J.; Medeiros, F. P. M.; Bieber, L. W.; Mendonça Junior, F. J. B.; Quim. Nova 2008, 31, 413.

22. Pettit, G. R.; Houghton, L. E.; Can. J. Chem. 1968, 46, 2471.

23. http://orgchem.colorado.edu/Technique/Procedures/Columnchrom/ Procedure.html, acessada em Janeiro 2013. 
PREPARAÇÃO DE DERIVADOS DO LAPACHOL EM MEIO ÁCIDO E EM MEIO BÁSICO: UMA PROPOSTA DE EXPERIMENTOS PARA A DISCIPLINA DE QUÍMICA ORGÂNICA EXPERIMENTAL

Ticiano Pereira Barbosa* e Hermes Diniz Neto

Faculdade de Ciências Médicas da Paraíba, Campus II, Praça de São Francisco, 16, 58010-650 João Pessoa - PB, Brasil

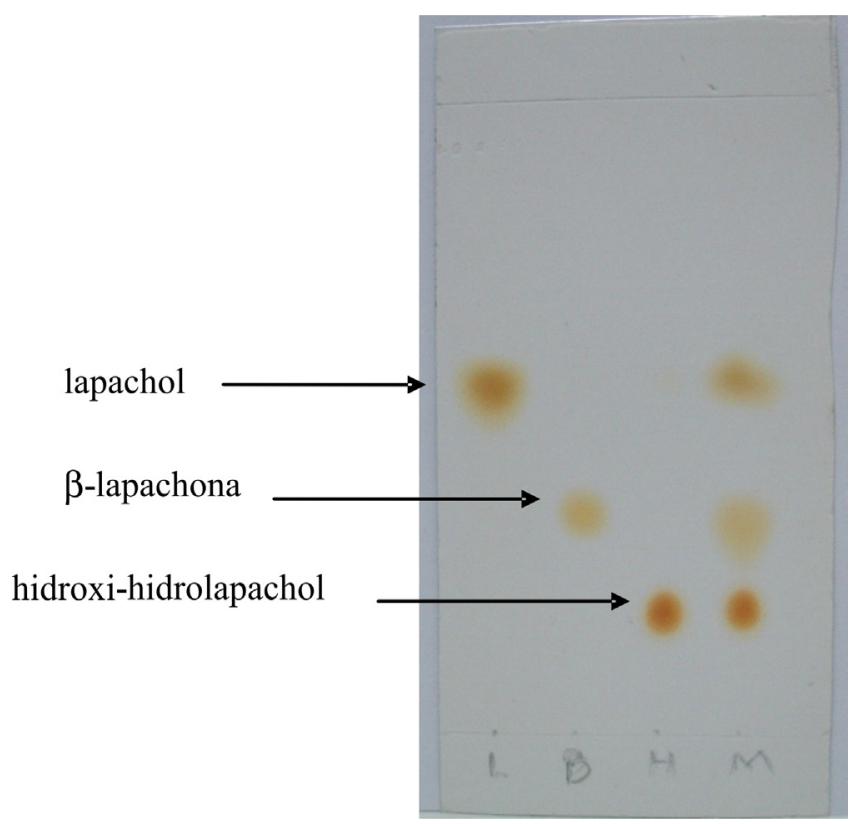

Figura 1S. CCD com as naftoquinonas utilizadas. Sistema eluente utilizado: acetato de etila/n-hexano (3:7)

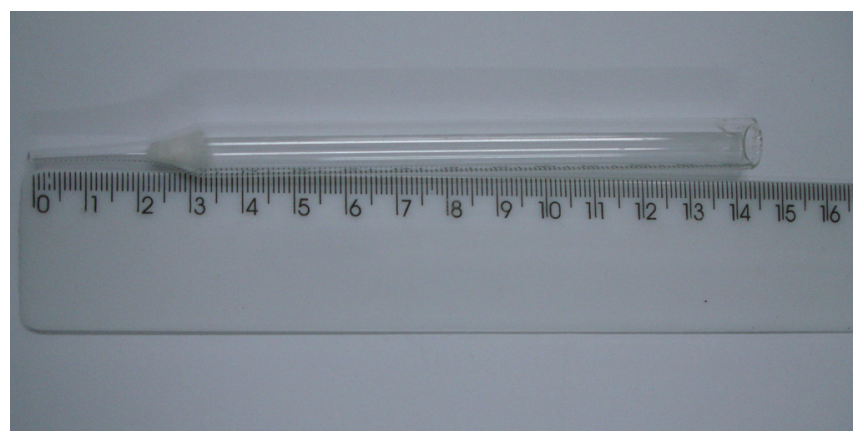

Figura 2S. Pipeta Pasteur adaptada para cromatografia em coluna com escala reduzida 